\title{
Retrospective Study of 23 Cases of Psoriasis Association with HIV Infection Observed in the Department of Dermatology-STD in the University Hospital of Donka Conakry Guinea
}

\author{
M. Keita1, M. M. Soumah¹, T. M. Tounkara1, D. Sylla², B. F. Diané1, F. B. Sako³, H. Baldé1, M. Cissé1 \\ ${ }^{1}$ Department of Dermatology-STD in the University Hospital of Donka, Conakry, Guinea \\ ${ }^{2}$ Department of Intensives Cares, Medico-Surgeon Urgences in the University Hospital of Donka, Conakry, Guinea \\ ${ }^{3}$ Department of Tropical Infection Diseases in the University Hospital of Donka, Conakry, Guinea \\ Email: medsoum7@yahoo.fr
}

How to cite this paper: Keita, M., Soumah, M.M., Tounkara, T.M., Sylla, D., Diané, B.F., Sako, F.B., Baldé, H. and Cissé, M. (2018) Retrospective Study of 23 Cases of Psoriasis Association with HIV Infection Observed in the Department of Dermatology-STD in the University Hospital of Donka Conakry Guinea. Journal of Cosmetics, Dermatological Sciences and Applications, 8, 1-5.

https://doi.org/10.4236/jcdsa.2018.81001

Received: November 27, 2017

Accepted: January 22, 2018

Published: January 25, 2018

Copyright $(9) 2018$ by authors and Scientific Research Publishing Inc. This work is licensed under the Creative Commons Attribution International License (CC BY 4.0).

http://creativecommons.org/licenses/by/4.0/

\begin{abstract}
Introduction: The psoriasis is one of the inflammatory dermatoses with unknown etiology, with chronic evolution having episodic appearance and disappearance. Its prevalence in HIV patients varied from $2 \%$ to $5 \%$ in most of the times. Objective of this study was to describe the demographic, clinical and therapeutic characteristics of psoriasis patients infected with HIV. Methods: It is a descriptive retrospective study done from January 2003 to December 2013 based on the information from the hospital card of hospitalized patients and outpatients taken care in the department of Dermatology-STD for psoriasis at the University hospital center in Donka Conakry. We included all the cases of psoriasis associated with HIV infection diagnosed from clinical and paraclinical elements. Results: We recorded 23 (24.73\%) cases of psoriasis associated with HIV infection among 93 patients observed for psoriasis in which there are 4 cases of psoriasis vulgaris, 10 cases of erythrodermic psoriasis and 9 cases of arthropathic form among these numbers. We had 7 women and 16 men. The medium age of our patients was $44.5 \pm 12$ years [27 - 62 years]. The delayed duration time of consultation varied from 30 to more than 180 days. The psoriasis was the circumstance of the discovery of the HIV infection among $55 \%$ of cases. The pruritus was the functional sign which is the most frequent in 20 cases among 23 cases and $71.4 \%$ of cases were accompanied with pain. Family history was found in $7.10 \%$ of cases. Anxiety was the dominant factor cause in $42 \%$ and the infection $38 \%$. The cutaneous alteration was noted in all patients; $92.9 \%$ of patients had nails alteration and intertrigineous association in $78.6 \%$ of cases. The clinical forms found were psoriasis
\end{abstract}


vulgaris $4 / 23$ cases, arthropathic psoriasis $9 / 23$ cases, erythrodermic psoriasis $10 / 23$. More than half (13/23) cases of our patients were diagnosed stage III of the classification of WHO. The complicated forms like erythrodermic and arthropathic psoriasis were frequent in patients whose total CD $4<350$ cells $/ \mu \mathrm{l}$ about $65 \%$. The most frequently encountered co-morbidity was tuberculosis (9 cases). The use of traditional therapeutic means was noted in $50 \%$ of cases. The local treatment was based on dermocorticoid and keratolylic drugs. The general treatment received by all patients was antiretroviral medication and Methotrexate. Discussion: Our results are of course not representing all the cases of psoriasis in Guinea but it gives us an idea of the importance of HIV and psoriasis association and the influence of immunodepression inducted by HIV during the evolution of psoriasis. The demographic, clinical and therapeutic characteristics described in our patients were near those reported by more authors. Conclusion: HIV-associated psoriasis does not appear to be familial. Serious clinical forms occur in highly immunocompromised patients.

\section{Keywords}

Psoriasis, HIV Infection, Epidemiology, Clinical, Therapeutic, Guinea

\section{Introduction}

The psoriasis is one of the inflammatory erythemato-squamous dermatosis with unknown etiology, having chronic evolution with episodes of thrust and remission more or less long [1]. In most cases, psoriasis is a benign disease that is uncomfortable with its unattractive appearance and the difficulty of all its life living with this pathology [2]. However, certain clinical forms are disabling, notably the arthropathic and erythrodermic forms [1] [2]. Its prevalence among HIV-infected individuals ranges from $2 \%$ to $5 \%$ in most series [2] [3]. The objective of this study was to describe in our context, the demographic, clinical and therapeutic characteristics of psoriatic patients infected with HIV.

\section{Methods}

This was a descriptive retrospective study of 11 years conducted from January 2003 to December 2013 from records of patients in hospital or outpatients for psoriasis in the department of Dermatology-STD of the University Hospital of Conakry. The study consisted of identifying and documenting all cases of HIV-associated psoriasis observed in the department. Data collection was done from a previously established survey sheet. The variables studied were: frequency of psoriasis and HIV association, usual demographic data (age, sex, community group), clinical data (family history of psoriasis, clinical signs, lesion topography, clinical forms, opportunistic infections, comorbidity, WHO stage of HIV infection [4] and treatment data). We did not use any of the usual severity scores used to measure the severity of psoriasis and the evaluation of treatment proto- 
cols. All cases of HIV-associated psoriasis diagnosed on the basis of clinical and/or paraclinical elements of both sexes were included in the study regardless of the clinical form of psoriasis and the clinical stage of HIV infection and regardless of the origin of the patients.

\section{Results}

We found 23 cases of psoriasis associated with HIV infection in 93 patients seen for psoriasis at a frequency of $24.73 \%$. These are 16 men and 7 women. The average age was $44.5 \pm 12$ years with extremes of 27 and 62 years. The consultation period ranged from less than 30 days to more than 180 days. Psoriasis was the circumstance of discovery of HIV infection in the majority of cases (55\%). Pruritus was the most common functional sign (16 cases/23 cases) and was accompanied by pain in $71.4 \%$ of cases.

The family history of psoriasis was found in $7.10 \%$ of cases. Triggers were dominated by stress (42\%) and infection (38\%). Cutaneous involvement (100\%) was noted in all patients; $92.9 \%$ of patients had associated phanterian involvement; intertriginous involvement was associated in $78.6 \%$ of cases. The clinical forms encountered were psoriasis vulgaris (4 cases or $17.39 \%$ ), erythrodermic psoriasis (10 cases or $3.48 \%$ ) and arthropathic psoriasis (9 cases or $39.13 \%$ ). More than half of the patients ( 13 cases) were in stage III of WHO. Complicated forms (erythrodermic and arthropathic psoriasis) were common in patients with CD4 counts $<350$ cells $/ \mu$ l $(65 \%)$. The most common comorbidity was tuberculosis (9 cases). The use of prior therapy was noted in half (50\%) of the cases. Local treatment was based on topical corticosteroids and keratolytics. The systemic treatment included the administration of antiretrovirals, oral retinoids and metothrexate, which allowed total bleaching of the patients.

\section{Discussion}

We conducted a retrospective descriptive study based on the records of psoriatic patients infected with HIV. The retrospective nature and the fact that the diagnosis of psoriasis in the majority of cases was made solely on the basis of clinical arguments constituted the limits of this study. The results obtained cannot be exhaustive, because the study only took into account the cases recorded in the reference department for the management of dermatological conditions. They cannot represent all psoriasis patients in Guinea, but give an idea of the profile of the association between psoriasis and HIV in Conakry.

Indeed, since the first description of a combination psoriasis and HIV in 1985, where it was noted that HIV infection could trigger or worsen known psoriasis, several other cases have been reported [5] [6]. The frequency of $24.73 \%$ in our study remains higher than that reported by Obuch [7] in San Francisco (2.5\%). In Berlin Wölfer et al. [8] report a frequency of 5\%, three times higher than in the general population. However, no link between the mode of transmission of the infection and the development of psoriatic lesions has been demonstrated. 
The average age of our patients is close to that of Dubertret et al. [1], who report that the age of psoriatic patients infected with HIV appears to be higher (36 years). Several studies [7] [8] report an early age of onset of psoriasis in women, but this is not universally observed. There is no evidence of morphological differences in this dermatosis between men and women [9]. In our series, psoriasis presents an inequality between the two sexes. HIV-infected men are twice tendacy to develop psoriasis than women.

Mebazaa et al. [10] stated in their study that recent onset or worsening of old psoriasis should lead to suspicion of immunosuppression and have to be rule out for HIV serology. This assertion is confirmed by our study where psoriasis was the circumstance of discovery of seropositivity in the majority of cases (55\%). The spontaneous appearance of psoriasis in $93.90 \%$ of our patients is identical to the observation of Mebazaa et al. [10] who report that 2/3 of his psoriatic patients infected with HIV had no history of psoriasis. Go BK et al. [11] in Singapore report that out of $96 \mathrm{HIV}$-infected patients, $70 \%$ of patients developed lesions at the time of diagnosis, suggesting that HIV has a direct effect on the onset of psoriasis. This association is probably related to the overexpression of tumor necrosis factor- $\alpha$ (TNF- $\alpha$ ) and interferon- $\gamma$ (IFN- $\gamma$ ) [11] [12].

As in the Leal L [5] and Manki I [13] series, the predominant clinical forms were erythrodermic psoriasis (10 cases) and arthropathic psoriasis (9 cases). HIV-induced immunosuppression would explain the preponderance of these complicated forms because most patients had CD4 $<350$ cells/ $\mu$ l. As for tuberculosis, its association is not fortuitous because it constitutes the first opportunistic pulmonary infection observed in people infected with HIV in Africa [14]. Immunomodulatory therapies usually indicated in psoriasis may exacerbate immunosuppression in these subjects and may interact with other drugs [15]. The choice of treatment must therefore take into account the benefit-risk ratio of the prescribed drug. Recently, "The National Psoriasis Foundation" has issued recommendations for the management of psoriasis in HIV-infected patients [10]. It offers first-line topical treatment (corticosteroids or topical vitamin D or combination vitamin D corticosteroids or keratolytics) for mild to moderate psoriasis and narrow-spectrum phototherapy associated with antiretrovirals in severe psoriasis. Oral retinoids (acitretin) are indicated as second-line therapy. For refractory cases, ciclosporin, metothrexate, or TNF- $\alpha$ therapy are offered at the cost of careful clinical and laboratory monitoring [15]. In our patients, the use of topical agents and the general treatment (metothrexate, retinoid) associated with antiretrovirals, allowed the fast and total bleaching of patients without significant side effects have been observed.

\section{Conclusion}

HIV-associated psoriasis does not appear to be familial. Serious clinical forms occur in highly immunocompromised patients. The recent onset or aggravation of old psoriasis should lead to suspicion of immunosuppression and HIV serology. 


\section{References}

[1] Dubertret, L. (1995) Psoriasis. ISED, Edition du Dome, 265-270.

[2] Gabla, A., Eti, E., Nebavi, Z., Aka, B., Kanga, J.M., Ouattara, B., et al. (1997) Le rhumatisme psoriasique en milieu hospitalier: A propos de 10 cas. Rhumatologie, 49, 91-94.

[3] Mahé, A., Bobin, P., Coulibaly, S. and Tounkara, A. (1997) Dermatoses révélatrices de l'infection par le virus de l'immunodéficience humaine au Mali. Annales De Dermatologie Et De Venereologie, 124, 144-150.

[4] Classification (2009) OMS/2004 in Mémento thérapeutique du VIH /SIDA en Afrique. 2ème édition.

[5] Leal, L., Ribera, M. and Dauden, E. (2008) Psoriasis and HIV Infection. Actas Dermo-Sifiliográficas, 99, 753-763. https://doi.org/10.1016/S0001-7310(08)74955-X

[6] Mallon, E. and Bunker, C.B. (2000) HIV-Associated Psoriasis. AIDS Patient Care STDs, 14, 239-246. https://doi.org/10.1089/108729100317696

[7] Obuch, M.L., Maurer, T.A., Becker, B. and Berger, T.G. (1992) Psoriasis and Human Immunodeficiency Virus Infection. Journal of the American Academy of Dermatology, 27, 667-673. https://doi.org/10.1016/0190-9622(92)70234-7

[8] Lu, W., Djemadji-Oudjiel, N. and Hiletework, M. (1998) HIV-assoziierte Psoriasis. Klinische und histologische Beobachtungen an 36 Patienten. Hautarzt, 49, 197-202.

[9] Farber, E.M. and Nall, L. (1998) Epidemiology: Natural History and Genetics. In: Roenigk Jr., H.H. and Maibach, H.I., Eds., Psoriasis, Dekker, New York, 107-115.

[10] Mebazaa, A., Hawilo, A., Abdelmalek, R., CheikhRouhou, R., Zribi, H. and Trojjet, S, (2011) Psoriasis révélant une infection par le VIH. Revue Tunisienne d'Infectiologie, 5, 36-38.

[11] Goh, B.K., Chan, R.K.W., Sen, P., Theng, C.T.S., Tan, H.H., Wu, Y.J., et al. (2007) Spectrum of Skin Disorders in Human Immunodeficiency Virus-Infected Patients in Singapore and the Relationship to CD4 Lymphocyte Counts. International Journal of Dermatology, 46, 695-699. https://doi.org/10.1111/j.1365-4632.2007.03164.x

[12] Ettehadi, P., Greaves, M.W., Wallach, D., Aderka, D. and Camp, R.D. (1994) Elevated Tumor Necrosis Factor-Alpha (TNF-Alpha) Biological Activity in Psoriatic Lesions. Clinical \& Experimental Immunology, 96, 146-151.

https://doi.org/10.1111/j.1365-2249.1994.tb06244.x

[13] Mamkin, I., Mamkin, A. and Ramanan, S. (2007) HIV-Associated Psoriasis. The Lancet Infectious Diseases, 7, 496. https://doi.org/10.1016/S1473-3099(07)70161-5

[14] Rosenheim, M., M'pele, P., Itoua-Ngaporo, A., Brucker, G., Duflo, B. and Gentilini, M. (1988) Propositions pour une stratégie diagnostique et thérapeutique de l'infection à VIH en Afrique. Méd Afr Noire, 34, 1021-1030.

[15] Menon, K., Van Voorhees, A.S., Bebo Jr., B.F., Gladman, D.D., Hsu, S., Kalb, R.E., et al. (2010) Psoriasis in Patients with HIV Infection: From the Medical Board of the National Psoriasis Foundation. Journal of the American Academy of Dermatology, 62, 291-299. https://doi.org/10.1016/j.jaad.2009.03.047 